\title{
Search For PeVatrons In VHE Gamma Rays And Neutrinos
}

\author{
Silvia Celli ${ }^{1, a)}$ \\ ${ }^{1}$ Gran Sasso Science Institute, Viale Francesco Crispi 7, 67100, L'Aquila, Italy. \\ a)Corresponding author: silvia.celli@gssi.infn.it
}

\begin{abstract}
Since its discovery more than one hundred years ago, the origin of the cosmic-ray (CR) flux measured on Earth is still unknown: in order to explain the region below the knee, supernova remnants (SNRs) are usually addressed as PeV cosmic accelerators. In particular, young SNRs are potential candidates since they might act as PeVatrons at least during the initial stage of their evolution. However, no clear indication of $\mathrm{PeV}$ energies has been observed so far in such a kind of sources, including the brightest TeV SNR, RX J1713-3946.7. Recently, the Galactic Center region has been detected as a very-high-energy (VHE) gamma-ray emitter. Two emission regions have been resolved by H.E.S.S.: a point source, spatially associated to the known radio source SgrA*, and a diffuse flux, characterised by a simple power law gamma-ray spectrum with no visible cut-off up to gammaray energies of about $35 \mathrm{TeV}$. Such a detection triggers the search for PeVatron at the center of our Galaxy. A clear evidence of the hadronic nature of the emission would be the detection of a neutrino counterpart. I will here discuss the potentials of the next generation neutrino telescopes.
\end{abstract}

\section{INTRODUCTION}

CRs are relativistic charged particles that reach the Earth with extremely high energies. They provide striking evidence of the existence of effective accelerators in the Universe, motivating the search for the astrophysical sources where they are produced. Below the so-called ankle at $E \simeq 3 \times 10^{17} \mathrm{eV}$, CRs are most likely confined by the magnetic field of the Milky Way, while particles with higher energy are probably originated outside our Galaxy. Since CRs are electrically charged, they cannot point back to their emission site, except in the ultra high energy domain, beyond the ankle, where however the particle flux is extremely low (1 particle per $\mathrm{km}^{2}$ per sr per year) to be suitable for astronomical purposes. On the other hand, photons constitute ideal messengers, being neutral and abundantly produced in sources through both leptonic and hadronic processes, where the former involve primary electrons while the latter involve primary hadrons. However, the large cross section of electromagnetic processes constitutes a limiting factor for high energy photons, since they can be absorbed in the interaction with interstellar radiation field, generating electromagnetic cascades. For instance, the omnipresent Cosmic Microwave Background (CMB) represents the main photon target for gamma rays in the channel of electron-positron pair production: this process is very effective for PeV gamma rays, whose horizon is consequently reduced to less than $10 \mathrm{kpc}$. Thus, gamma rays are very effective in directly probing the galactic scale. At larger energies, neutrinos constitute the only astronomical messenger able to unveil distant particle accelerators. However, the small cross section of weak interaction combined with the low neutrino fluxes expected from known high energy sources makes the case for cubic kilometer detectors in order to collect about $10-100$ astrophysical events per year, on top of a large atmospheric background. To this extent, a huge effort has been devoted in the last 30 years to the development of such instruments: currently, the deep waters of the Mediterranean Sea are equipped with the 12-strings operational ANTARES telescope [1], while the South Pole ice is instrumented with the 86-strings IceCube Observatory [2]. Since 2013, extra-terrestrial neutrinos have been observed by IceCube in the form of a diffuse flux [3]: the origin of such a flux are still unknown, thus demanding for a deep and careful investigation. Recently, a bulk of neutrinos from the flaring blazar TXS 0506+056 was announced [4], opening the era of neutrino astronomy. In the following Section, I will review the experimental technique devoted to neutrino detection and the current status of observations. Then, I will focus on two candidate galactic CR accelerators, namely SNRs and the Galactic Center region, in view of their possible neutrino counterpart. As I will discuss through the paper, cubic kilometer class telescopes in the Northern hemisphere have the potential of observing high-energy neutrinos from these important astronomical objects and can thus check the existence of hadronic PeV Galactic accelerators. 


\section{NEUTRINO TELESCOPES}

High-energy neutrino telescopes are three dimensional arrays of photomultipliers, where Cherenkov radiation induced by neutrino interaction products is observed: the position, time and charge deposit are used to infer both the direction and the energy of the incoming neutrino. Although neutrino detectors are sensitive to all neutrino flavors, from the point of view of the reconstruction of arrival directions of primary neutrinos, the best channel is represented by neutrinos producing muons. The main background source for this event sample is then constituted by atmospheric muons and the accompanying atmospheric neutrinos. In order to reduce atmospheric muons, events coming from below the detector horizon are selected (the so called upward going sample), as background muons are absorbed in their path through the Earth. Alternatively, also events coming from above the detector horizon can be used, selecting a reduced fiducial volume of the detector. This is possible for high-energy starting events (HESE), where atmospheric muons are used in order to veto the accompanying atmospheric neutrinos. The same containment technique allows to include cascade events to neutrino searches: the usage of this additional channel allows to significantly increase the sample statistics, given the full sky coverage of this event sample. The IceCube detector has validated the search strategy for the detection of a neutrino signal [5]: as shown in Fig. 1, the through-going muon sample collected in eight years of data-taking shows a $6.7 \sigma$ evidence for an astrophysical flux described by an unbroken power law as

$$
\frac{d \Phi}{d E}\left(v_{\mu}+\bar{v}_{\mu}\right)=\left(1.01 \pm_{0.23}^{0.26}\right)\left(\frac{E}{100 \mathrm{TeV}}\right)^{-2.19 \pm 0.10} \times 10^{-18} \mathrm{GeV}^{-1} \mathrm{~cm}^{-2} \mathrm{~s}^{-1} \mathrm{sr}^{-1}
$$

while the HESE sample collected in six years of data at energies larger than $60 \mathrm{TeV}$ shows a neutrino flux as steep as

$$
\frac{d \Phi}{d E}(v+\bar{v})_{H E S E}=(2.46 \pm 0.8)\left(\frac{E}{100 \mathrm{TeV}}\right)^{-2.92} \times 10^{-18} \mathrm{GeV}^{-1} \mathrm{~cm}^{-2} \mathrm{~s}^{-1} \mathrm{sr}^{-1}
$$

Still nowadays, the origin of such a signal is unknown, as well as the reason for the through-going muon spectrum being considerably harder than that from HESE. It was pointed out that part of the detected flux might have been originated in the Galactic Plane. Further investigation of this scenario is necessary: a more extended statistical sample is required to test different interpretation hypotheses, which is going to be provided by the next generation of neutrino telescopes. In particular, a telescope in the Northern Hemisphere, as the under construction KM3NeT [6] will provide a high visibility of the Galactic Plane through the clean event sample from muon neutrinos. In the following, I will discuss the potentials for a neutrino detection from Galactic sources by the next generation of multi- $\mathrm{km}^{3}$ instruments (see [7] for further insight into the topic).

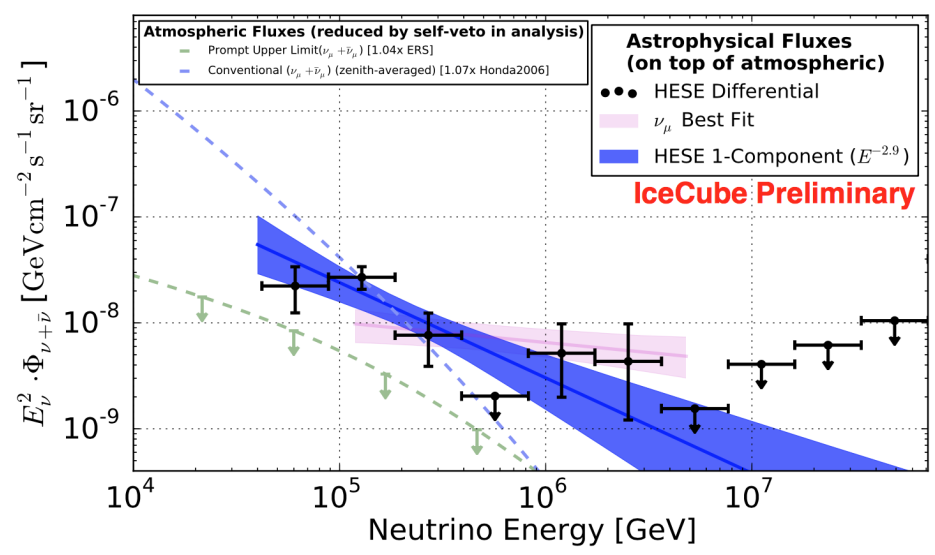

FIGURE 1. IceCube per flavor neutrino flux (combined neutrino and anti-neutrino) as a function of energy [5]. The black points have $1 \sigma$ uncertainties. Atmospheric fluxes are already subtracted, while a fit to the charm component is shown separately. The blue band shows the $1 \sigma$ uncertainty on the HESE flux, while the pink band refers to the through-going muons. 


\section{SUPERNOVA REMNANTS AND THE PARADIGM ON THE ORIGIN OF GALACTIC COSMIC RAYS}

Though CRs have been discovered about a century ago, the question about their origin is still a matter of discussion. Baade and Zwicky [8] have been the first to propose that supernovae might be the sources of CRs, and this still remains the most popular scenario to explain the origin of Galactic CRs. The particle energy that marks the transition between galactic and extra-galactic CRs is believed to be located between the so-called knee (at $E \simeq 3 \times 10^{15} \mathrm{eV}$ ) and the ankle. Thus, the acceleration mechanism connected to supernovae must be able to accelerate particles up to the PeV energy range and beyond. The present formulation of this idea is often referred to as the SNR paradigm for the origin of CRs. The success of this paradigm relies on several facts: i) SNRs can provide the power needed to sustain the CR flux at the observed level, if some fraction (about 10\%) of their kinetic energy is converted into relativistic particles, ii) diffusive shock acceleration can operate at SNR shocks and this provides a viable mechanism to accelerate CRs, and iii) during the acceleration process CRs can amplify the magnetic field at shocks via various plasma instabilities and consequently the magnetic field strength at SNR shocks might grow up to a level that allows the acceleration of particles to PeV energies. All of these considerations support the idea that SNRs indeed are the sources of CRs, though an unambiguous and conclusive proof of such a statement is still missing. The acceleration of CRs must be accompanied by the production of gamma rays. The detection of several SNRs in TeV gamma rays nicely fits within the paradigm predictions, but it cannot be considered a proof of the fact that SNRs can accelerate CRs. This is because electrons can as well be accelerated at shocks, and their inverse Compton emission on the $\mathrm{CMB}$ radiation field can also account for the observed $\mathrm{TeV}$ radiation. Thus the ambiguity between the hadronic or leptonic origin of the gamma-ray emission observed from SNRs is the main obstacle in proving (or falsifying) the SNR paradigm for the origin of CRs. Multi-wavelength observations of SNRs, from the radio band to the VHE gamma-ray domain, can help in solving such a degeneracy. This is generally done on a case by case basis, given the large diversity in SNR spectra, as shown in Fig. 2(a) for the known TeV emitters. In some cases, it has been possible to ascribe quite confidently the gamma-ray emission either to a hadronic or a leptonic mechanism, while for other cases the situation still remains ambiguous.

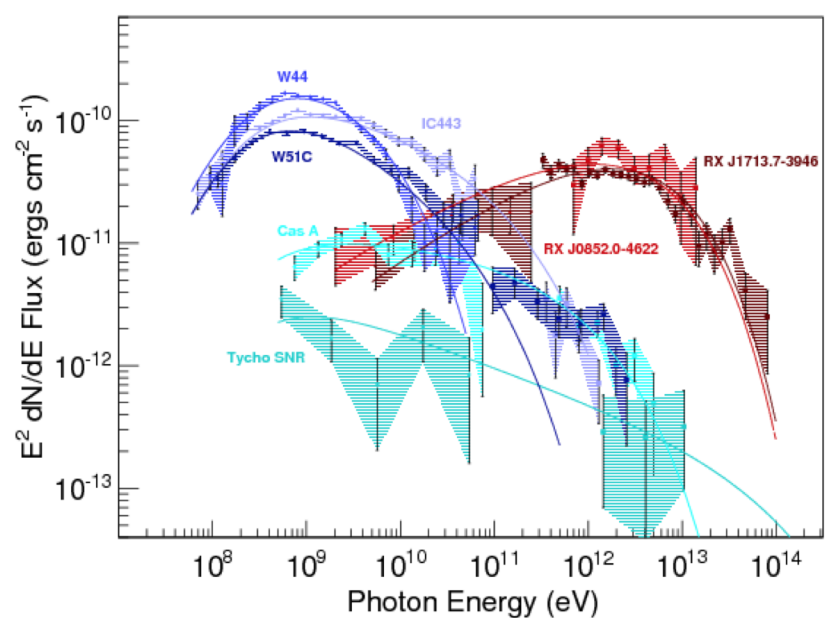

(a)

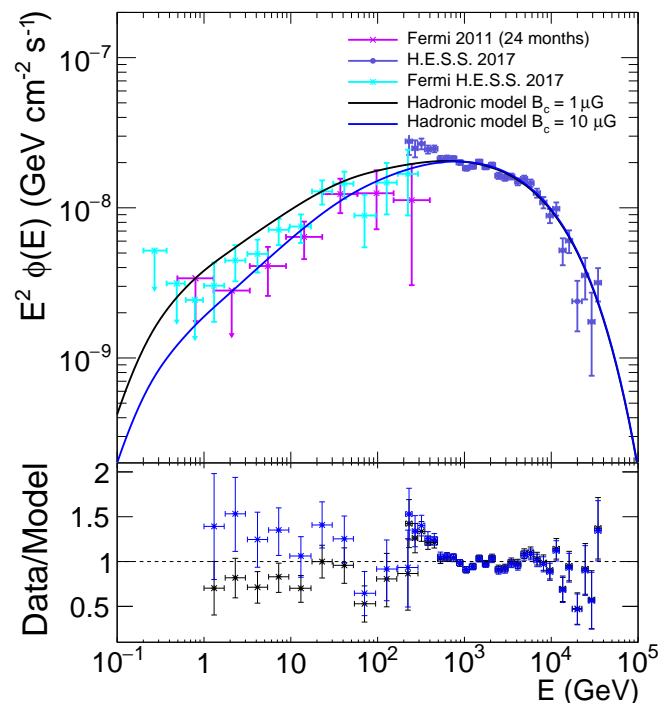

(b)

FIGURE 2. Left: Energy spectra of the most prominent SNRs and the correspondent hadronic fits to the data (solid lines) [9]. Right: Gamma-ray flux from the SNR RX J1713.7-3946. The data points refer respectively to: i) 24 months of Fermi-LAT data (magenta), ii) H.E.S.S. data (violet), and iii) H.E.S.S. analysis of five years of Fermi-LAT data (light blue). The hadronic models (solid lines) refer to a clumpy scenario, with different configurations of the magnetic field $B_{c}$ inside the clump (the black line stands for $B_{c}=1 \mu \mathrm{G}$ while the blue one for $\left.B_{c}=10 \mu \mathrm{G}\right)[10]$. 
This is for instance the case for RX J1713.7-3946: this remnant has been considered for long time the best candidate for an efficient acceleration scenario, mainly due to its high gamma-ray flux. The detection of gamma-ray emission in the energy range $[1-300] \mathrm{GeV}$ by the Fermi-LAT satellite has shown an unusually hard spectrum which, at a first glance, seems to be in a better agreement with a leptonic scenario. Nevertheless, a deeper analysis shows that neither the hadronic nor the leptonic scenarios, taken in their simplest form, can unequivocally explain the observations. To address this issue, it has been proposed [11] that the very hard energy spectrum at low energies could result also from hadronic emission if the SNR is expanding inside a clumpy medium. In such a case, in fact, hydrodynamical instabilities resulting from the interactions among the shock and dense clumps can amplify the magnetic field all around the clump, making difficult for particles at low energies to penetrate, and therefore interact, inside the proton target. Consequently, the resulting gamma-ray spectrum would be harder than the parent proton spectrum accelerated at the forward shock. A detail numerical simulation of such a scenario was presented in [10], where moreover a consistent modeling of the remnant $\mathrm{GeV}-\mathrm{TeV}$ emission was proposed in the context of a clumpy hadronic scenario, as illustrated in Fig. 2(b). A conclusive answer on the hadronic or leptonic origin of the observed radiation awaits the detection of neutrinos: a sensitivity down to $10^{-12} \mathrm{TeV} \mathrm{cm}^{-2} \mathrm{~s}^{-1}$ in the $10 \mathrm{TeV}$ domain is necessary to access the neutrino flux expected from this source. Such a value is within the reach of KM3NeT project in less than 10 years of operation.

\section{THE GALACTIC CENTER REGION}

The supermassive black-hole in the center of the Milky Way, located in correspondence of the radio source Sgr A*, is one of the most interesting astronomical objects. It is now in a state of relative inactivity, nonetheless it might be a non stationary source: as an example, Fermi bubbles could be visible manifestations of its past activity. The latest observations by the H.E.S.S. observatory [12], that various regions around Sgr A* emit gamma rays till many tens of $\mathrm{TeV}$ (see Fig. 3), offer new occasions to investigate this object. The excess of VHE gamma rays reported comes from two regions around the Galactic Center: a point source (HESS J1745-290), identified by a circular region centered on the radio source $\operatorname{Sgr} \mathrm{A}^{*}$ with a radius of $0.1^{\circ}$, and a diffuse emission, coming from an annulus with inner and outer radii of $0.15^{\circ}$ and $0.45^{\circ}$ respectively (20-63 pc). While the point source emission shows a cut-off at energies of about $10 \mathrm{TeV}$ in gamma rays, the diffuse gamma rays extend till very high energies $(\sim 35 \mathrm{TeV})$ without a perceivable cut-off: the respective energy spectra are reported in Fig. 5(a). A likely hypothesis is that the gamma rays seen by H.E.S.S. can be attributed to CR collisions: in this case, a PeV CR accelerator would be located right in the center of our Galaxy, a so-called PeVatron.
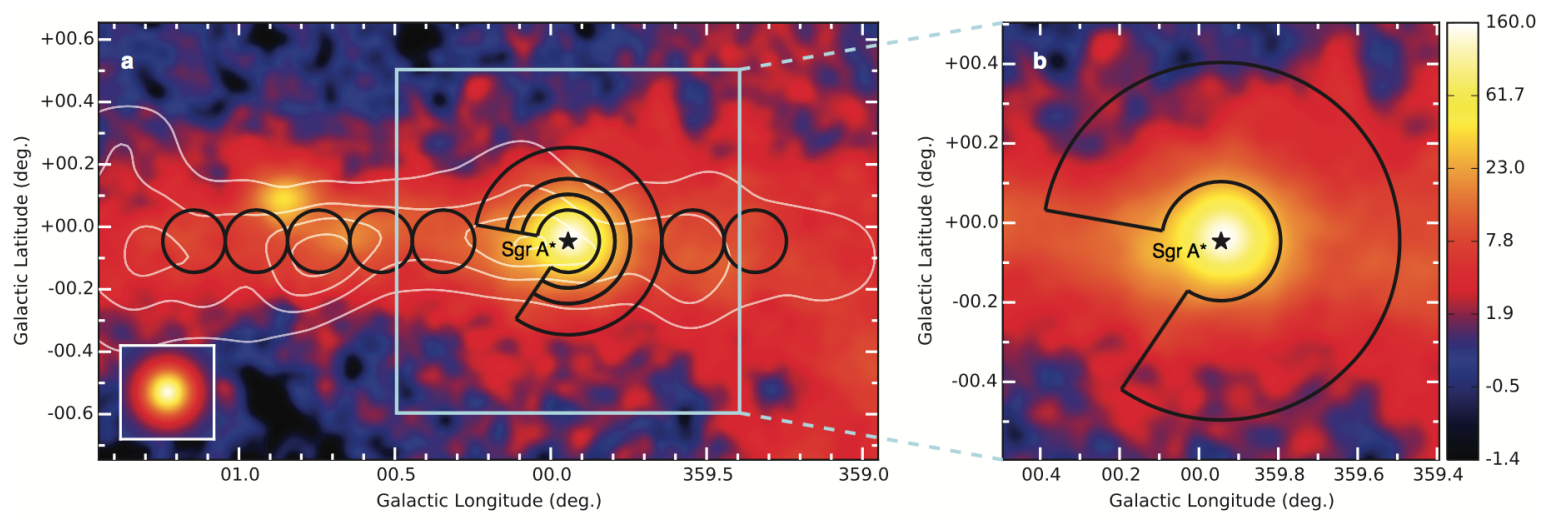

FIGURE 3. H.E.S.S. VHE gamma-ray image of the Galactic Centre region and zoomed view of the inner $\sim 70 \mathrm{pc}[12]$.

A crucial piece of information in the PeVatron scenario is constituted by the radial distribution of CRs in the observed region. Indeed, the CR radial profile and the distribution of the target gas in the central molecular zone shape the morphology of the VHE gamma-ray emission. Fig. 4 shows the radial profile of the $E \geq 10 \mathrm{TeV}$ CR energy density $w_{C R}$ up to $r \sim 200 \mathrm{pc}$, as derived through the observed gamma-ray luminosity and distribution of target gas. Such a CR density in the central molecular zone is found to be an order of magnitude larger than that of the 'sea' of 
CRs that universally fills the Galaxy, while the energy density of low energy (GeV) CRs in this region has a level comparable to it. This requires the presence of one or more accelerators of multi-TeV particles operating in the central molecular zone. Moreover, the radial distribution is compatible with a $w(r) \sim 1 / r$ profile, as shown in Fig. 4: this is expected in the case of a continuous injection of particles, which are then subject to diffusion into the Galaxy. The $1 / r$ profile of the CR density up to $200 \mathrm{pc}$ indicates a quasi-continuous injection of protons into the central molecular zone from a centrally located accelerator on a timescale $\Delta t$ exceeding the characteristic time of diffusive escape of particles from the central molecular zone, i.e. $\Delta t \geq 2 \times 10^{3} \mathrm{yr}$ for a typical diffusion coefficient of multi-TeV CRs of $D=10^{30} \mathrm{~cm}^{2} \mathrm{~s}^{-1}[12]$.

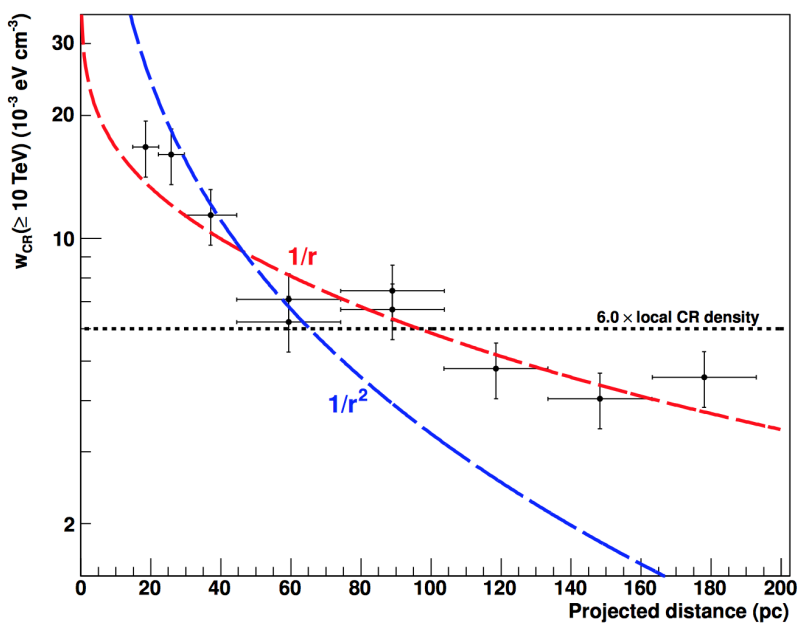

FIGURE 4. Radial distribution of CR energy density, for particles with $E>10 \mathrm{TeV}$ located in the region around Sgr A* [12].

The proof of the correctness of the PeVatron hypothesis requires neutrino telescopes. In this connection, it is essential to derive reliable predictions for the search of a neutrino signal from Sgr A* and its surroundings. When dealing with high-energy photons, one should always take into account the effect of absorption in their propagation from the source to the Earth, due to the presence of background radiation fields of the Milky Way. Thus, considering the observed gamma-ray spectrum, the corresponding emission spectrum is larger: this is obtained by modeling and then by removing the effect of absorption (de-absorption). The neutrino spectrum corresponds to the emission spectrum, and thus it is larger than the one obtained by converting the observed gamma-ray spectrum instead. Considering the known background radiation fields, as the CMB, the star light (SL) in optical frequencies and the infrared (IR) field, marginal effects are expected at the energies currently probed by H.E.S.S.; however, it is not possible to exclude larger effects due to non-standard radiation fields located near the very center. This would imply that the cut-off observed in the point source emission would be a manifestation of the absorption process rather than a feature connected to the maximum energy of the accelerator. A detailed procedure to characterize the radiation field required to explain a cut-off at around $E_{\gamma} \sim 10 \mathrm{TeV}$ was presented in [13]. In this scenario, the background radiation field that causes absorption is characterized by a temperature $T=1.3 \times 10^{-2} \mathrm{eV}$ and a column density of $L \times n_{\gamma}=1.5 \times 10^{25}$ ph cm $\mathrm{cm}^{-2}$. This is illustrated in the right panel of Fig. 5(a): the curve called 'PS fit with non standard abs.' is a power law spectrum with the same spectral index and normalization of the point source observed by H.E.S.S., that is modified taking into account the above scenario for gamma-ray absorption. Such a non standard IR radiation field could be produced in the reprocessing of the radiation from the central source, due to collision with circumnuclear disk clumps. Moreover, the field is supposed to be very much localized in the central region so that the diffuse component would not affected by it. Finally, in order to determine observationally whether the cut-off is intrinsic to the source or it is an absorption feature, measurements of gamma rays at energies above tens of $\mathrm{TeV}$ are required: this discrimination will be achieved by the next generation of imaging atmospheric Cherenkov telescopes, as CTA [14]. Assuming that the observed gamma rays are fully hadronic, it is possible to derive a theoretical upper bound on the expected neutrino emission from this region. In the following several different emitting regions are considered: i) a point source with a spectrum as that observed by H.E.S.S., that is a power law with an intrinsic cut-off connected to the acceleration mechanism located around $E_{\gamma} \sim 10 \mathrm{TeV}$, ii) a point source where the observed cut-off is connected with an absorption feature while the 


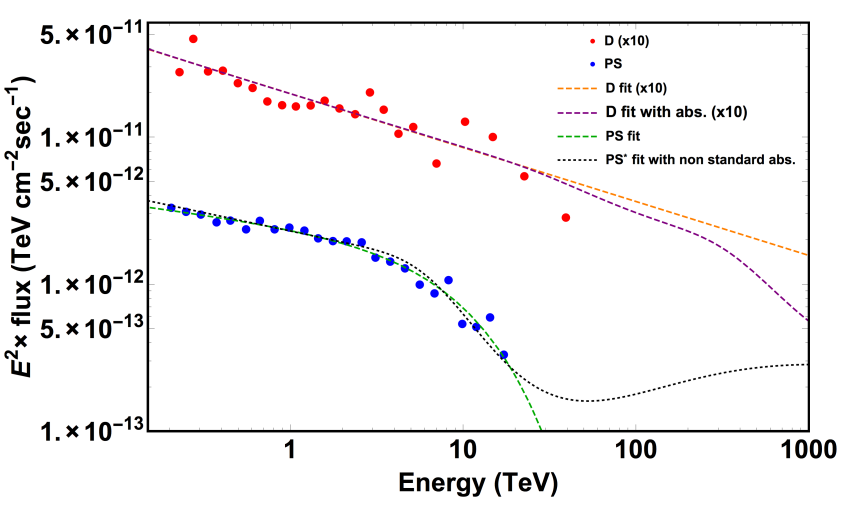

(a)

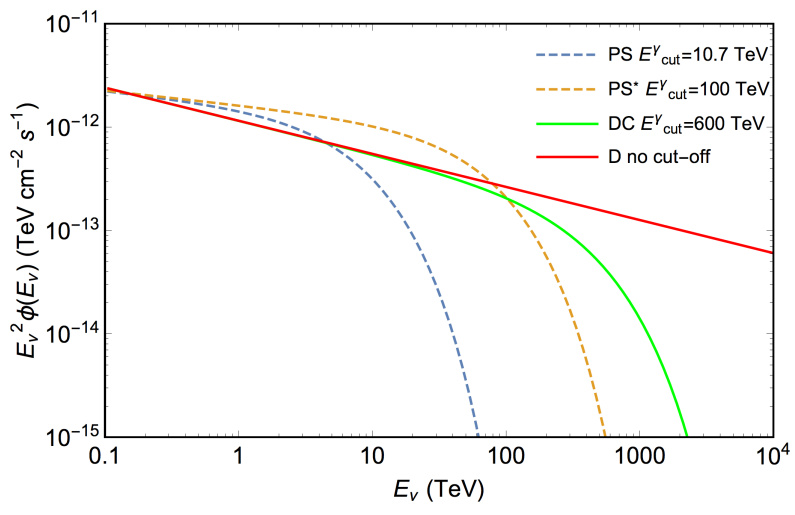

(b)

FIGURE 5. Left: Fits of the H.E.S.S. data: D is the diffuse flux, PS is the point source flux. For D, absorption due to CMB, IR and SL is considered. For PS, an increased absorption, due to non standard radiation field, is considered. Right: Muon neutrino fluxes expected from the Galactic Center region [13].

intrinsic cut-off is located at $E_{\gamma} \sim 100 \mathrm{TeV}$, iii) a diffuse emission without any cut-off and iv) a diffuse emission with a cut-off located at $E_{\gamma} \sim 600 \mathrm{TeV}$. The predicted muon neutrino spectra correspondent to these hypotheses are shown in Fig. 5(b). The expected rate of events in current neutrino telescopes ANTARES and IceCube is in the best case of the order of $0.1 v_{\mu}$ /year: such a low value has not allowed until now to probe any of the proposed scenarios. However, this rate would improve by one order of magnitude already with a cubic kilometer detector in the Northern Hemisphere: the expected rates of muon neutrinos in the ARCA infrastructure (the initial implementation of KM3NeT with a volume will be comparable to that of IceCube) are respectively i) $1 v_{\mu}$ /year in the standard point source scenario, ii) $2 v_{\mu}$ /year in the point source scenario assuming gamma-ray absorption and iii) $1.5 v_{\mu}$ /year from the diffuse region. This improvement is connected to few main features of KM3NeT: i) being located in the water, its angular resolution is significantly better with respect to instruments settled in the ice, allowing for a better background rejection; ii) being located at a larger deepness in the water, the amount of atmospheric muon background is substantially reduced and finally iii) being located in the Northern Hemisphere, it observes the Galactic Center region through a clear sample of upgoing muon tracks, allowing the detector to access a lower energy threshold with respect to the HESE sample. The last point is particularly relevant in the case of gamma-ray sources with an energy cut-off in the $1-100 \mathrm{TeV}$ region. This is indeed the energy region where most of the neutrino signal is expected. Nonetheless, in this energy band, neutrino telescopes can not avoid the issue of atmospheric neutrino background events. Thus, several years of data-taking will be required in order to establish the presence of a PeV proton Galactic accelerator and address the origin of Galactic CRs. In case of non-detection, however, strong constraints will be derived concerning the proton acceleration efficiency of this poorly-understood source.

\section{REFERENCES}

[1] ANTARES Collaboration, Nucl. Instrum. Meth. in Phys. Res. A 656, 11-38 (2011), arXiv:1104.1607 . IceCube Collaboration, Astroparticle Physics 26, 155-173 (2006), astro-ph/0604450 . IceCube Collaboration, Science 342, p. 1242856 (2013), arXiv:1311.5238 .

[4] IceCube Collaboration, Science (2018), 10.1126/science.aat2890.

[5] IceCube Collaboration, ArXiv e-prints (2017), arXiv:1710.01191 .

[6] KM3NeT Collaboration, Journal of Physics G Nuclear Physics 43, p. 084001 (2016), arXiv:1601.07459 .

[7] L. Ambrogi, S. Celli, and F. Aharonian, Astroparticle Physics 100, 69-79 (2018), arXiv:1803.03565 .

[8] W. Baade and F. Zwicky, Proceedings of the National Academy of Science 20, 259-263 (1934).

[9] S. Funk, Annual Review of Nuclear and Particle Science 65, 245-277 (2015), arXiv:1508.05190 . S. Celli, G. Morlino, S. Gabici, and F. Aharonian, ArXiv e-prints (2018), arXiv:1804.10579 .

[12] H.E.S.S. Collaboration, Nature 531, p. 476 (2016), arXiv:1603.07730 .

[13] S. Celli, A. Palladino, and F. Vissani, European Physical Journal C 77, p. 66 (2017), arXiv:1604.08791 .

[14] CTA Consortium, Proceedings RICH 2013, Nucl. Instrum. Meth. A766, 73-77 (2014), arXiv:1405.5696 . 